Kragujevac Journal of Mathematics

Volume 38(1) (2014), Pages 73-81.

\title{
POTENTIALLY GRAPHIC SEQUENCES OF SPLIT GRAPHS
}

\author{
S. PIRZADA ${ }^{1}$ AND BILAL A. CHAT ${ }^{2}$
}

\begin{abstract}
A sequence $\pi=\left(d_{1}, d_{2}, \ldots, d_{n}\right)$ of non-negative integers is said to be graphic if it is the degree sequence of a simple $G$ on $n$ vertices, and such a graph $G$ is referred to as a realization of $\pi$. The set of all non-increasing non-negative integer sequences $\pi=\left(d_{1}, d_{2}, \ldots, d_{n}\right)$ is denoted by $N S_{n}$. A sequence $\pi \in N S_{n}$ is said to be graphic if it is the degree sequence of a graph $G$ on $n$ vertices, and such a graph $\mathrm{G}$ is called a realization of $\pi$. The set of all graphic sequences in $N S_{n}$ is denoted by $G S_{n}$. A split graph $K_{r}+\overline{K_{s}}$ on $r+s$ vertices is denoted by $S_{r, s}$. A graphic sequence $\pi$ is potentially $H$-graphic if there is a realizaton of $\pi$ containing $H$ as a subgraph. In this paper, we determine the graphic sequences of subgraphs $H$, where $H$ is $S_{r_{1}, s_{1}}+S_{r_{2}, s_{2}}+S_{r_{3}, s_{3}}+\ldots+S_{r_{m}, s_{m}}, S_{r_{1}, s_{1}} \vee S_{r_{2}, s_{2}} \vee \ldots \vee S_{r_{m}, s_{m}}$ and $S_{r_{1}, s_{1}} \times S_{r_{2}, s_{2}} \times \ldots \times S_{r_{m}, s_{m}}$ and,$+ V$ and $\times$ denotes the standard join operation, the normal join operation and the cartesian product in these graphs respectively.
\end{abstract}

\section{INTRODUCTION}

Let $G$ be an undirected simple graph (graph without multiple edges and loops) with $n$ vertices and $m$ edges having vertex set $V(G)=\left\{v_{1}, v_{2}, \ldots, v_{n}\right\}$. Any undefined notations follows that of Bondy and Murty [1]. Throughout the paper, we denote such a graph by $G(n, m)$. The set of all non-increasing non-negative integer sequences $\pi=\left(d_{1}, d_{2}, \ldots, d_{n}\right)$ is denoted by $N S_{n}$. There are several famous results, Havel and Hakimi [5, 6] and Erdös and Gallai [3] which give necessary and sufficient conditions for a sequence $\pi=\left(d_{1}, d_{2}, \ldots, d_{n}\right)$ to be the degree sequence of a simple graph $G$. Unfortunately, knowing that a sequence has a realization gives no information about the properties that such a graph might have. In this paper, we explore this question of properties of a graph which is related to work originally introduced by A. R. Rao [9]. A sequence $\pi \in N S_{n}$ is said to be graphic if it is the degree sequence of a simple graph $G$ on $n$ vertices, and such a graph $G$ is called a realization of $\pi$. The sequence

Key words and phrases. Graph, Split graph, Potentially H-graphical sequences.

2010 Mathematics Subject Classification. Primary: 05C07.

Received: November, 19, 2013

Revised: October 24, 2013. 
$\pi=\left(d_{1}, d_{2}, \ldots, d_{n}\right)$ is graphic if and only if the sequence $\pi^{\prime}$ obtained from $\pi$ by laying off an element is graphic [7]. Also $d^{r_{1} \times r_{2}}$ means $d$ occurs $r_{1} \times r_{2}$ times in $\pi$. The set of all graphic sequences in $N S_{n}$ is denoted by $G S_{n}$. A graphic sequence $\pi$ is potentially $H$-graphic if there is a realization of $\pi$ containing $H$ as a subgraph, while $\pi$ is forcibly $H$ graphic if every realization of $\pi$ contains $H$ as a subgraph. If $\pi$ has a realization in which the $r+1$ vertices of largest degree induce a clique, then $\pi$ is said to be potentially $A_{r+1}$-graphic. The graphic sequence $\pi$ is potentially $K_{k+1}$-graphic if and only if $\pi$ is potentially $A_{k+1}$-graphic [10]. Let $\sigma(\pi)=d_{1}+d_{2}+\ldots+d_{n}$. If $G$ and $G_{1}$ are graphs, then $G \bigcup G_{1}$ is the disjoint union of $G$ and $G_{1}$. If $G=G_{1}$, we abbreviate $G \bigcup G_{1}$ as $2 G$. We denote $G+H$ as the graph with $V(G+H)=V(G) \bigcup V(H)$ and $E(G+H)=E(G) \bigcup E(H) \bigcup\{x y: x \in V(G), y \in V(H)\}$. Let $K_{k}, C_{k}, T_{k}$ and $P_{k}$ respectively denote a complete graph on $k$ vertices, a cycle on $k$ vertices, a tree on $k+1$ vertices and a path on $k+1$ vertices. Let $F_{k}$ denote the friendship graph on $2 k+1$ vertices, that is, the graph of $k$ triangles intersecting in a single vertex. For $0 \leq r \leq t$, denote the generalized friendship graph on $k t-k r+r$ vertices by $F_{t, r, k}$, where $F_{t, r, k}$ is the graph of $k$ copies of $K_{t}$ meeting in a common $r$ set.

Given a graph $H$, what is the maximum number of edges of a graph with $n$ vertices not containing $H$ as subgraph? This number is denoted by ex $(n, H)$, and is known as the Turan number. In terms of graphic sequences, the number $2 e x(n, H)+2$ is the minimum even integer $l$ such that every $n$-term graphic sequence $\pi$ with $\sigma(\pi) \geq l$ is forcibly $H$-graphic. Erdös, Jacobson and Lehel [2] first considered the following variant: determine the minimum even integer $l$ such that every $n$-term graphic sequence $\pi$ with $\sigma(\pi) \geq l$ is potentially $H$-graphic. We denote this minimum $l$ by $\sigma(H, n)$. A sequence $\pi=\left(d_{1}, d_{2}, \ldots, d_{n}\right)$ is said to be potentially $K_{r+1}$-graphic if there is a realization $G$ of $\pi$ containing $K_{r+1}$ as a subgraph. If $\pi$ is a graphic sequence with a realization $G$ containing $H$ as a subgraph, then there is a realization $G^{\prime}$ of $\pi$ containing $H$ with the vertices of $H$ having $|V(H)|$ largest degree of $\pi$ [4]. Let $S_{r, s}=K_{r}+\bar{K}_{s}$ be split graph on $r+s$ vertices, where $\overline{K_{s}}$ is the complement of $K_{s}$ and + denotes the standard join operation. As seen in [11, $S_{r, 1}=K_{r+1}$ and so the graph $S_{r, s}$ is an extension of the graph $K_{r+1}$. A sequence $\pi=\left(d_{1}, d_{2}, \ldots, d_{n}\right)$ is said to be potentially $S_{r, s^{-}}$graphic if there is a realization $G$ of $\pi$ containing $S_{r, s}$ as a subgraph. Yin Jain Hua and Haikou [11] obtained a Havel-Hakimi type procedure and a simple sufficient condition for $\pi$ to be potentially $S_{r, s}$-graphic. We have the following definitions.

Definition 1.1. 8] For the graphs $G_{1}, G_{2}$ with disjoint vertex set $V\left(G_{1}\right), V\left(G_{2}\right)$ the cartesian product is a graph $G=G_{1} \times G_{2}$ with vertex set $V\left(G_{1}\right) \times V\left(G_{2}\right)$ and an edge $\left(\left(u_{1}, v_{1}\right),\left(u_{2}, v_{2}\right)\right)$ iff $u_{1}=u_{2}$ and $v_{1} v_{2}$ is an edge of $G_{2}$.

Definition 1.2. [11] The standard join of $S_{r_{1}, s_{1}}, S_{r_{2}, s_{2}}$ is a graph $S=S_{r_{1}, s_{1}} \vee S_{r_{2}, s_{2}}$ with vertex set $V\left(S_{r_{1}, s_{1}}\right) \cup V\left(S_{r_{2}, s_{2}}\right)$ and an edge set consisting of all edges of $S_{r_{1}, s_{1}}$ and $S_{r_{2}, s_{2}}$ together with the edges joining each vertex of $K_{r_{1}}$ of $S_{r_{1}, s_{1}}$ with every vertex of $S_{r_{2}, s_{2}}$ and $s_{1}$ vertices of $S_{r_{1}, s_{1}}$ are joined with only vertices of $K_{r_{2}}$ in $S_{r_{2}, s_{2}}$. 
Definition 1.3. [8] The join ( complete product) of $G_{1}$ and $G_{2}$ is a graph $G=G_{1} \vee G_{2}$ with vertex set $V\left(G_{1}\right) \cup V\left(G_{2}\right)$ and an edge set consisting of all edges of $G_{1}$ and $G_{2}$ together with the edges joining each vertex of $G_{1}$ with every vertex of $G_{2}$.

Definition 1.4. [9] The split graph $K_{r}+\overline{K_{s}}$ on $r+s$ vertices is denoted by $S_{r, s}$ where + denotes the standard join operation and $\overline{K_{s}}$ is the complement of $K_{s}$. A non-increasing sequence $\pi=\left(d_{1}, d_{2}, \ldots, d_{n}\right)$ of non-negative integers is said to be potentially $S_{r, s^{-}}$ graphic if there exists a realization $G$ of $\pi$ containing $S_{r, s}$ as a subgraph.

Definition 1.5. If $\pi$ has a realization $G$ containing $K_{r+1}$ on those vertices having degree $d_{1}, d_{2}, \ldots, d_{r+1}$, then $\pi$ is potentially $A_{r+1}$-graphic.

Definition 1.6. 10] The tensor product (conjuction), denoted by $G=G_{1} \wedge G_{2}$, is the graph with vertex set $V=V_{1} \times V_{2}$ and for any two vertices $w_{1}=\left(u_{1}, v_{1}\right)$ and $w_{2}=\left(u_{2}, v_{2}\right)$ in $V ; u_{1}, u_{2} \in V_{1}$ and $v_{1}, v_{2} \in V_{2}$, there is an edge $w_{1} w_{2} \in E(G)$ if and only if $u_{1} u_{2} \in E_{1}$ and $v_{1} v_{2} \in E_{2}$.

\section{MAIN RESUlts}

We start with the following result.

Theorem 2.1. If $\pi_{1}=\left(d_{1}^{1}, d_{2}^{1}, \ldots, d_{m}^{1}\right)$ is potentially $K_{p_{1}}$-graphic and $\pi_{2}=$ $\left(d_{1}^{2}, d_{2}^{2}, \ldots, d_{n}^{2}\right)$ is potentially $K_{p_{2}}$-graphic, $p_{1} \leq m$ and $p_{2} \leq n$, then the graphic sequence $\pi$ of $G=G_{1} \times G_{2}$ is potentially $p_{1}+p_{2}-2$ regular graphic.

Proof. Let $\pi_{1}=\left(d_{1}^{1}, d_{2}^{1}, \ldots, d_{m}^{1}\right)$ and $\pi_{2}=\left(d_{1}^{2}, d_{2}^{2}, \ldots, d_{n}^{2}\right)$ be respectively $K_{p_{1}}$-graphic and $K_{p_{2}}$-graphic. Then there exists graphs $G_{1}$ and $G_{2}$ respectively realizing $\pi_{1}$ and $\pi_{2}$ and respectively containing $K_{p_{1}}$ and $K_{p_{2}}$ as subgraphs. Let $G=G_{1} \times G_{2}$ be the cartesian product of $G_{1}$ and $G_{2}$ and let $\pi_{3}=\left(d_{11}, d_{12}, \ldots, d_{1 m}, d_{21}, d_{22}, \ldots, d_{2 m}, \ldots, d_{m 1}\right.$, $\left.d_{m 2}, \ldots, d_{m n}\right)$ be the graphic sequence of $G_{1} \times G_{2}$. Then $d_{i j}=d_{i}^{1}+d_{j}^{2}$ for $1 \leq i \leq m$ and $1 \leq j \leq n$ where $d_{i j}$ is the degree of $i j^{\text {th }}$ vertex in $G$. We have to show that the realization $G$ of $\pi$ contains $p_{1}+p_{2}-2$ as a regular subgraph. To prove this, it is enough to show that sum of degrees of this subgraph is equal to $p_{1} p_{2}\left(p_{1}+p_{2}-2\right)$. Clearly,

$$
\sum_{i=1}^{m} \sum_{j=1}^{n} d_{i j}=\sum_{i=1}^{m} \sum_{j=1}^{n} d_{i}^{1}+d_{j}^{2}=\left(d_{1}^{1}+d_{1}^{2}\right)+\ldots+\left(d_{1}^{1}+d_{n}^{2}\right)+\ldots+\left(d_{m}^{1}+d_{n}^{2}\right) .
$$

This is true for all $m$ and $n$. In particular, it holds for $m=p_{1}$ and $n=p_{2}$. Therefore

$$
\begin{aligned}
\sum_{i=1}^{p_{1}} \sum_{j=1}^{p_{2}} d_{i j}= & \left(d_{1}^{1}+d_{1}^{2}\right)+\left(d_{1}^{1}+d_{2}^{2}\right)+\ldots+\left(d_{1}^{1}+d_{p_{2}}^{2}\right)+\ldots+\left(d_{p_{1}}^{1}+d_{p_{2}}^{2}\right) \\
= & \left(p_{1}-1+p_{2}-1\right)+\left(p_{1}-1+p_{2}-1\right)+\ldots+\left(p_{1}-1+p_{2}-1\right) \\
& +\ldots+\left(p_{1}-1+p_{2}-1\right) \\
= & p_{1} p_{2}\left(p_{1}+p_{2}-2\right) .
\end{aligned}
$$


Theorem 2.1 can be generalized as follows.

Theorem 2.2. If $\pi_{i}=\left(d_{1}^{i}, d_{2}^{i}, \cdots, d_{n_{j}}^{i}\right)$ is potentially $K_{p_{i}}$-graphic for $i, j=1,2, \ldots, r$ with $p_{i} \leq n_{j}$, then the graphic sequence $\pi$ of $G=G_{1} \times G_{2} \times \ldots \times G_{r}$ is a potentially $\sum_{i=1}^{r} p_{i}-r$ regular graphic.

Proof. The proof follows by induction on $r$.

Theorem 2.3. If $\pi_{1}=\left(d_{1}^{1}, d_{2}^{1}, \ldots, d_{m}^{1}\right)$ is potentially $K_{p_{1}}$-graphic and $\pi_{2}=$ $\left(d_{1}^{2}, d_{2}^{2}, \ldots, d_{n}^{2}\right)$ is potentially $K_{p_{2}}$-graphic, $p_{1} \leq m$ and $p_{2} \leq n$, then the graphic sequence $\pi$ of $G=G_{1}+G_{2}$ is potentially $K_{p_{1}+p_{2}}$-graphic.

Proof. Let $\pi_{1}=\left(d_{1}^{1}, d_{2}^{1}, \ldots, d_{m}^{1}\right)$ be potentially $K_{p_{1}}$-graphic. Then there exists a graph $G_{1}$ which realizes $\pi_{1}$ and will contain $K_{p_{1}}$ as a subgraph. Let $\pi_{2}=\left(d_{1}^{2}, d_{2}^{2}, \ldots, d_{n}^{2}\right)$ be potentially $K_{p_{2}}$ - graphic, so there exists a graph $G_{2}$ which realizes $\pi_{2}$ and will contain $K_{p_{2}}$ as a subgraph. Let $G=G_{1}+G_{2}$ be the join of $G_{1}$ and $G_{2}$ and let $\pi=\left(d_{1}, d_{2}, \ldots, d_{m+n}\right)$ be the graphic sequence of $G=G_{1}+G_{2}$. Then we have

$$
\begin{array}{r}
d_{i}=d_{i}^{1}+n \text { for } i=1,2, \ldots, m \\
d_{m+j}=d_{j}^{2}+m \text { for } j=1,2, \ldots, n .
\end{array}
$$

We have to show that the realization of $\pi$ contains $K_{p_{1}+p_{2}}$ as a subgraph. To prove this it is enough to show that

$$
\sum_{i=1}^{p_{1}} d_{i}+\sum_{j=1}^{p_{2}} d_{m+j}=\left(p_{1}+p_{2}\right)\left(p_{1}+p_{2}-1\right) .
$$

We take the summation to the equations in 2.1) respectively from $i=1,2, \ldots, m$ and $j=1,2, \ldots, n$ and get $\sum_{i=1}^{m} d_{i}=\sum_{i=1}^{m} d_{i}^{1}+\sum_{i=1}^{m} n$ and $\sum_{j=1}^{m} d_{m+j}=\sum_{j=1}^{n} d_{j}^{2}+\sum_{j=1}^{n} m$. These two equations imply

$$
\sum_{i=1}^{m} d_{i}=\sum_{i=1}^{m} d_{i}^{1}+m n
$$

and

$$
\sum_{j=1}^{m} d_{m+j}=\sum_{j=1}^{n} d_{j}^{2}+n m
$$

As 2.2 and (2.3) is true for all $m$ and $n$, therefore, in particular it is true for $m=p_{1}$ and $n=p_{2}$. So, 


$$
\begin{aligned}
\sum_{i=1}^{p_{1}} d_{i}+\sum_{i=1}^{p_{2}} d_{m+j} & =\sum_{i=1}^{p_{1}} d_{i}^{1}+\sum_{i=1}^{p_{2}} d_{m+j}^{2}+2 p_{1} p_{2} \\
& =d_{1}^{1}+d_{2}^{1}+\ldots+d_{p_{1}}^{1}+d_{1}^{2}+d_{2}^{2}+\ldots+d_{p_{2}}^{2}+2 p_{1} p_{2} \\
& =\left(p_{1}-1\right)+\ldots+\left(p_{1}-1\right)+\left(p_{2}-1\right)+\ldots+\left(p_{2}-1\right)+2 p_{1} p_{2} \\
& =p_{1}\left(p_{1}-1\right)+p_{2}\left(p_{2}-1\right)+p_{1} p_{2}+p_{1} p_{2} \\
& =p_{1}\left(p_{1}+p_{2}-1\right)+p_{2}\left(p_{2}+p_{1}-1\right) \\
& =\left(p_{1}+p_{2}\right)\left(p_{1}+p_{2}-1\right) .
\end{aligned}
$$

Theorem 2.3 can be generalized as follows.

Theorem 2.4. If $\pi_{i}=\left(d_{1}^{i}, d_{2}^{i}, \ldots, d_{n_{j}}^{i}\right)$ is potentially $K_{p_{i}}$-graphic for $i=1,2, \ldots, r$ with $p_{i} \leq n_{j}$. Then the graphic sequence $\pi$ of $G=G_{1}+G_{2}+\ldots+G_{r}$ is potentially $K_{\sum_{i=1}^{r} p_{i}}$-graphic.

Proof. This can be proved by induction on $r$.

Theorem 2.5. If $\pi_{i}$ is potentially $S_{r_{i}, s_{i}}$-graphic for $i=1,2, \ldots, m$, then

(1) The graphic sequence $\pi$ of $G=G_{1}+G_{2}+\ldots+G_{m}$ is potentially $S_{\sum_{i=1}^{m} r_{i}, \sum_{i=1}^{m} s_{i}}$-graphic, where + denotes the standard join operation in $S_{r_{i}, s_{i}}$.

(2) The graphic sequence of $S_{\sum_{i=1}^{m} r_{i}, \sum_{i=1}^{m} s_{i}}$ for $j=1,2, \ldots, m$ is

$$
\pi^{\prime}=\left(\left(\sum_{i=1}^{m}\left(r_{i}+s_{i}-1\right)\right)^{r_{j}},\left(\sum_{i=1}^{m} r_{i}\right)^{s_{j}}\right)
$$

(3) Also, $\sigma\left(\pi^{\prime}\right)=\left(\sum_{i=1}^{m} r_{i}\right)^{2}+2\left(\sum_{i=1}^{m} r_{i}\right)\left(\sum_{i=1}^{m} s_{i}\right)-\left(\sum_{i=1}^{m} r_{i}\right)$.

Proof. Let $\pi$ be potentially $S_{r_{i}, s_{i}}$-graphic for $i=1,2, \ldots, m$. Then there exists a graph $G_{i}$ which realizes $\pi_{i}$ and will contain $S_{r_{i}, s_{i}}$ as a subgraph. Let $G=G_{1}+G_{2}+\ldots+G_{m}$ be the graph obtained from $G_{1}, G_{2}, \ldots, G_{m}$ by using join operation. Therefore, clearly the graphic sequence $\pi$ of $G$ is potentially $S_{\sum_{i=1}^{m} r_{i}, \sum_{i=1}^{m} s_{i}}$-graphic follows from Theorem 2.4. To prove part (2), we use induction on $m$. For $k=1$, the result is obvious. For $k=2$, we have $G=G_{1}+G_{2}$. Therefore, in particular $S_{r_{1}+r_{2}, s_{1}+s_{2}}=S_{r_{1}, s_{1}}+S_{r_{2}, s_{2}}$. Now by Theorem 2.4, we have for every $i=1,2, \ldots, r_{1}$ and $i=1,2,3, \ldots, r_{2}$ and $j=1,2,3, \ldots, s_{1}$ and $j=1,2,3, \ldots, s_{2}$

$$
\overline{d_{i}}=d_{i}+r_{2}+s_{1}+s_{2}
$$


and

$$
\overline{d_{j}}=r_{1}+r_{2},
$$

where $\overline{d_{i}}$ and $\overline{d_{j}}$ are respectively the degree of $\overline{v_{i}^{t h}}$ and $\overline{v_{j}^{t h}}$ vertex in $S_{r_{1}+r_{2}, s_{1}+s_{2}}$ and $d_{i}$ is the degree of $i^{\text {th }}$ vertex in $K_{r_{1}}$. Equations (2.4) and (2.5) hold for every i, j. Thus

$$
\begin{aligned}
\pi^{2} & =\left(\left(r_{1}+r_{2}+s_{1}+s_{2}-1\right)^{r_{1}},\left(r_{1}+r_{2}+s_{1}+s_{2}-1\right)^{r_{2}},\left(r_{1}+r_{2}\right)^{s_{1}},\left(r_{1}+r_{2}\right)^{s_{2}}\right) \\
& =\left(\left(\sum_{i=1}^{2}\left(r_{i}+s_{i}\right)\right)^{r_{j}},\left(\sum_{i=1}^{2} r_{i}\right)^{s_{j}}\right) .
\end{aligned}
$$

This shows that the result is true for $k=2$. Assume that the result holds for $k=m-1$, therefore $\pi^{m-1}=\left(\left(\sum_{i=1}^{m-1}\left(r_{i}+s_{i}\right)\right)^{r_{j}},\left(\sum_{i=1}^{m-1} r_{i}\right)^{s_{j}}\right)$, for $j=1,2, \ldots, m-1$. Now for $k=m$ we have that $G=S_{r_{1}, s_{1}}+S_{r_{2}, s_{2}}+\ldots+S_{r_{m-1}, s_{m-1}}+S_{r_{m}, s_{m}}=A+S_{r_{m}, s_{m}}$, where $A=S_{r_{1}, s_{1}}+S_{r_{2}, s_{2}}+\ldots+S_{r_{m-1}, s_{m-1}}$.

Since the result is proved for every $k=m-1$ and using the fact that the result is proved for each pair and since the result is already proved for $k=2$, it follows by induction hypothesis that the result holds for $k=m$ also. That is,

$$
\pi=\pi^{m}=\left(\left(\sum_{i=1}^{m}\left(r_{i}+s_{i}-1\right)\right)^{r_{j}},\left(\sum_{i=1}^{m} r_{i}\right)^{s_{j}}\right) .
$$

This proves part (2). To prove part (3), we have for $j=1,2, \ldots, m$ that

$$
\begin{aligned}
\sigma\left(\pi^{\prime}\right) & =r_{j}\left(\sum_{i=1}^{m}\left(r_{i}+s_{i}-1\right)\right)+s_{j}\left(\sum_{i=1}^{m} r_{i}\right) \\
& =r_{j}\left(\sum_{i=1}^{m}\left(r_{i}+s_{i}\right)\right)-r_{j}+s_{j}\left(\sum_{i=1}^{m} r_{i}\right) \\
& =\sum_{j=1}^{m} r_{j}\left(\sum_{i=1}^{m}\left(r_{i}+s_{i}\right)\right)-\sum_{j=1}^{m} r_{j}+\sum_{j=1}^{m} s_{j} \sum_{i=1}^{m} r_{i} \\
& =\left(\sum_{i=1}^{m} r_{i}\right)^{2}+2\left(\sum_{i=1}^{m} r_{i}\right)\left(\sum_{i=1}^{m} s_{i}\right)-\left(\sum_{i=1}^{m} r_{i}\right) .
\end{aligned}
$$

Theorem 2.6. If $\pi_{1}=\left(d_{1}^{1}, d_{2}^{1}, \ldots, d_{m}^{1}\right)$ is potentially $S_{r_{1}, s_{1}}$-graphic and $\pi_{2}=$ $\left(d_{1}^{2}, d_{2}^{2}, \ldots, d_{n}^{2}\right)$ is potentially $S_{r_{2}, s_{2}}$-graphic. Then

(1) $\pi_{s_{1} \times s_{2}}$ of $S_{1} \times S_{2}$ is graphic,

(2) the graphic sequence of $S_{1} \times S_{2}$ is $\pi_{s_{1} \times s_{2}}=\left(d_{i j}^{r_{1} \times r_{2}}, d_{i j}^{r_{1} \times s_{2}}, d_{i j}^{s_{1} \times r_{2}}, d_{i j}^{s_{1} \times s_{2}}\right)$, where $d_{i j}$ is the degree of ijth vertex in $S_{1} \times S_{2}$. 
Proof. Let $\pi_{1}=\left(d_{1}^{1}, d_{2}^{1}, \ldots, d_{m}^{1}\right)$ be potentially $S_{r_{1}, s_{1}}$-graphic. Then there exists a graph $G_{1}$ which realizes $\pi_{1}$ and will contain $S_{r_{1}, s_{1}}$ as a subgraph. Let $\pi_{2}=$ $\left(d_{1}^{2}, d_{2}^{2}, \ldots, d_{n}^{2}\right)$ be potentially $S_{r_{2}, s_{2}}$ graphic so that there exists a graph $G_{2}$ which realizes $\pi_{2}$ and will contain $S_{r_{2}, s_{2}}$ as a subgraph. Let $G=G_{1} \times G_{2}$ be the cartesian product of $G_{1}$ and $G_{2}$. Then we have $d_{i j}=d_{i}+d_{j}$ for $1 \leq i \leq m$ and $1 \leq j \leq n$. This relation is true for every vertex of the graph $G$, therefore it also holds for the graph $S=S_{1} \times S_{2}$. Thus we can write $S=S_{r_{1}, s_{1}} \times S_{r_{2}, s_{2}}$. We have

$$
d_{i j}=d_{i}+d_{j} \quad \text { for } \quad 1 \leq i \leq r_{1}+s_{1} \quad \text { and } \quad 1 \leq j \leq r_{2}+s_{2} .
$$

for $1 \leq i \leq r_{1}+s_{1}$ and $1 \leq j \leq r_{2}+s_{2}$.

If $d_{i}$ is the degree of $i t h$ vertex of $r_{1}$ in $S_{r_{1}, s_{1}}$ and $d_{j}$ is the degree of $j$ th vertex of $r_{2}$ in $S_{r_{2}, s_{2}}$, it can be seen by construction that degree of $i j t h$ vertex of $r_{1} \times r_{2}$ in $\mathrm{S}$ is $d_{i j}$, where $d_{i j}$ is defined above and this term occurs in $r_{1} \times r_{2}$ in $\pi_{S_{1} \times S_{2}}$. Similarly other degree terms of the sequence occurs in $r_{1} \times s_{2}, s_{1} \times r_{2}, s_{1} \times s_{2}$ by using definition of cartesian product of graphs. Thus $\pi_{s_{1} \times s_{2}}=\left(d_{i j}^{r_{1} \times r_{2}}, d_{i j}^{r_{1} \times s_{2}}, d_{i j}^{s_{1} \times r_{2}}, d_{i j}^{s_{1} \times s_{2}}\right)$. This completes the proof of the theorem.

The following result is a generalization of Theorem 2.6 whose proof follows simply by induction.

Theorem 2.7. If $\pi_{i}=\left(d_{1}^{i}, d_{2}^{i}, \ldots, d_{n_{j}}^{i}\right)$ is potentially $S_{r_{i}, s_{i}}$-graphic, then

(1) the sequence $\pi$ of $G=S_{r_{1}, s_{1}} \times S_{r_{2}, s_{2}} \times \ldots \times S_{r_{m}, s_{m}}$ is graphic,

(2) the graphic sequence of $\pi$ is $\pi_{s_{1} \times s_{2} \times \ldots \times s_{m}}=\left(d_{i j k \ldots m}^{r_{1} \times r_{2} \times \ldots \times r_{m}}, d_{i j k \ldots m}^{r_{1} \times r_{2} \times \ldots \times r_{m-1} \times s_{m}}, \ldots\right.$, $\left.d_{i j k \ldots m}^{s_{1} \times s_{2} \times s_{3} \times \ldots \times s_{m-1} \times r_{m}}, \ldots, d_{i j k \ldots m}^{s_{1} \times s_{2} \times \ldots \times s_{m}}\right)$, where $d_{i j k \ldots m}=d_{i}+d_{j}+d_{k}+\ldots+d_{m}$.

Proof. This can be proved by induction on $r$.

Theorem 2.8. If $\pi_{i}$ is potentially $S_{r_{i}, s_{i}}$-graphic for $i=1,2, \ldots, m$, then

(1) the graphic sequence $\pi$ of $G=G_{1} \vee G_{2} \vee \ldots \vee G_{m}$ is potentially $S_{\sum_{i=1}^{m} r_{i}, \sum_{i=1}^{m} s_{i}}$ graphic, where $\vee$ denotes the join operation in $G_{1}, G_{2}, \ldots, G_{n}$,

(2) the graphic sequence of $S_{\sum_{i=1}^{m} r_{i}, \sum_{i=1}^{m} s_{i}}$ is

$$
\pi^{\prime}=\left(\left(\sum_{i=1}^{m}\left(r_{i}+s_{i}-1\right)\right)^{r_{j}},\left(\sum_{i=1}^{m} r_{i}+\sum_{i=1, i \neq j}^{m} s_{i}\right)^{s_{j}}\right), \text { for } j=1,2, \ldots, m
$$

(3) and $\sigma\left(\pi^{\prime}\right)=\left(\sum_{i=1}^{m} r_{i}\right)^{2}+2 \sum_{i=1}^{m} r_{i} \sum_{j=1}^{m} s_{j}+\left(\sum_{i=1}^{m} s_{i}\right)^{2}+\sum_{j=1}^{m} s_{j}\left(\sum_{i=1, i \neq j}^{m} s_{i}\right)-\sum_{i=1}^{m} r_{i}$.

Proof. Let $\pi$ be potentially $S_{r_{i}, s_{i}}$-graphic for $i=1,2, \ldots, m$. Then there exists a graph $G_{i}$ which realizes $\pi_{i}$ and will contain $S_{r_{i}, s_{i}}$ as a subgraph. Let $G=G_{1} \vee G_{2} \vee \ldots \vee G_{m}$ be the graph obtained from $G_{1}, G_{2}, \ldots, G_{m}$ by using join operation. Therefore, clearly the graphic sequence $\pi$ of $G$ is potentially $S_{\sum_{i=1}^{m} r_{i}, \sum_{i=1}^{m} s_{i}}$-graphic. 
To prove part (2), we use induction on $m$. For $k=1$, the result is obvious. For $k=2$, we have $G=G_{1} \vee G_{2}$, therefore, in particular we take the normal join operation between graphs $S_{r_{1}, s_{1}}$ and $S_{r_{2}, s_{2}}$. Thus we have $S_{1,2}=S_{r_{1}, s_{1}} \vee S_{r_{2}, s_{2}}$. Now by Theorem 2.6. we have for every $i=1,2, \ldots, r_{1}$ and $i=1,2,3, \ldots, r_{2}$ and $j=1,2,3, \ldots, s_{1}$ and $j=1,2,3, \ldots, s_{2}$

$$
\overline{d_{i}}=d_{i}+r_{2}+s_{1}+s_{2}
$$

and

$$
\overline{d_{j}}=r_{1}+r_{2}+s_{2},
$$

where $\overline{d_{i}}$ and $\overline{d_{j}}$ are respectively the degree of $\overline{v_{i}^{t h}}$ and $\overline{v_{j}^{t h}}$ vertex in $S_{r_{1}+r_{2}, s_{1}+s_{2}}$ and $d_{i}$ is the degree of $i^{t h}$ vertex in $K_{r_{1}}$. Equations (2.7) and (2.8) hold for every $i, j$. Thus for $j=1,2$

$$
\begin{aligned}
\pi^{2}= & \left(\left(r_{1}+r_{2}+s_{1}+s_{2}-1\right)^{r_{1}},\left(r_{1}+r_{2}+s_{1}+s_{2}-1\right)^{r_{2}}\right. \\
& \left.\left(r_{1}+r_{2}+s_{2}\right)^{s_{1}},\left(r_{1}+r_{2}+s_{1}\right)^{s_{2}}\right) \\
= & \left(\left(\sum_{i=1}^{2}\left(r_{i}+s_{i}-1\right)\right)^{r_{j}},\left(\sum_{i=1}^{2} r_{i}+\sum_{i=1, i \neq j}^{2} s_{i}\right)^{r_{j}}\right) .
\end{aligned}
$$

This shows that the result is true for $k=2$. Assume that the result holds for $k=m-1$, therefore $\pi^{m-1}=\left(\left(\sum_{i=1}^{m-1}\left(r_{i}+s_{i}-1\right)\right)^{r_{j}},\left(\sum_{i=1}^{m-1} r_{i}+\sum_{i=1, i \neq j}^{m-1} s_{i}\right)^{r_{j}}\right)$, for all $j=1,2, \ldots, m-1$. Now for $k=m$ we have that $G=S_{r_{1}, s_{1}} \vee S_{r_{2}, s_{2}} \vee \ldots \vee S_{r_{m-1}, s_{m-1}} \vee$ $S_{r_{m}, s_{m}}=A \vee S_{r_{m}, s_{m}}$, where $A=S_{r_{1}, s_{1}} \vee S_{r_{2}, s_{2}} \vee \ldots \vee S_{r_{m-1}, s_{m-1}}$.

Since the result is proved for all $k=m-1$ and using the fact that the result is proved for each pair and since the result is already proved for $k=2$, it follows by induction hypothesis that result holds for $k=m$ also. That is,

$$
\pi=\pi^{m}=\left(\left(\sum_{i=1}^{m}\left(r_{i}+s_{i}-1\right)\right)^{r_{j}},\left(\sum_{i=1}^{m} r_{i}+\sum_{i=1, i \neq j}^{m} s_{i}\right)^{s_{j}}\right) .
$$

This proves part (2). To prove part (3), we have for all $j=1,2, \ldots, m$

$$
\begin{aligned}
\sigma\left(\pi^{\prime}\right) & =r_{j}\left(\sum_{i=1}^{m}\left(r_{i}+s_{i}-1\right)\right)+s_{j}\left(\sum_{i=1}^{m} r_{i}+\sum_{i=1, i \neq j}^{m} s_{i}\right) \\
& =r_{j}\left(\sum_{i=1}^{m}\left(r_{i}+s_{i}\right)\right)-r_{j}+s_{j}\left(\sum_{i=1}^{m} r_{i}+\sum_{i=1, i \neq j}^{m} s_{j}\right)
\end{aligned}
$$




$$
=\left(\sum_{j=1}^{m} r_{i}\right)^{2}+2 \sum_{i=1}^{m} r_{i} \sum_{i=1}^{m} s_{i}+\sum_{i=1}^{m} s_{j}\left(\sum_{i=1, i \neq j}^{m} s_{i}\right)-\sum_{i=1}^{m} r_{i} .
$$

Remark 2.1. Let $\pi_{1}=\left(d_{1}^{1}, d_{2}^{1} \ldots, d_{m}^{1}\right)$ be potentially $K_{p_{1}}$-graphic $\pi_{2}=\left(d_{1}^{2}, d_{2}^{2} \cdots, d_{m}^{2}\right)$ be potentially $K_{p_{2}}$ graphic. Then the graphic sequence $\pi$ of $G=G_{1} \wedge G_{2}$ is potentially $H_{p}$-graphic, where $H_{p}$ is a $p$ - regular graph and $p$ depends upon $p_{1}$ and $p_{2}$. If $p_{1}=3$ and $p_{2}=2$, then $\pi$ of $G=G_{1} \wedge G_{2}$ is potentially $H_{2}$-graphic. If $p_{1}=3$ and $p_{2}=3$, then $\pi$ of $G=G_{1} \wedge G_{2}$ is potentially $H_{4}$-graphic. If $p_{1}=4$ and $p_{2}=4$, then $\pi$ of $G=G_{1} \wedge G_{2}$ is potentially $H_{9}$ graphic. If $p_{1}=3$ and $p_{2}=4$, then $\pi$ of $G=G_{1} \wedge G_{2}$ is potentially $H_{6}$-graphic. From this we conclude that $p$ depends upon $p_{1}$ and $p_{2}$.

\section{REFERENCES}

[1] J. A. Bondy and U. S. R. Murty, Graph Theory with Applications, The Macmillan Press Ltd, 1976.

[2] P. Erdos, M. S. Jacobson, J. Lehel, Graphs realising the same degree sequences and their respective clique numbers, Graph Theory, Combinatorics and Applications, 1 (1991) 439-449.

[3] P. Erdos and T. Gallai, Graphs with prescribed degrees (in Hungarian) Matematikai Lapok, 11 (1960) 264-274.

[4] R. J. Gould, M. S. Jacobson and J. Lehel, Potentially G-graphic degree sequences, Combinatorics, Graph Theory, and Algorithms, 1 (1999) 387-460.

[5] S. L. Hakimi, On the realizability of a set of Integers as degrees of the vertices of a graph, J. SIAM Appl. Math., 10 (1962) 496-506.

[6] V. Havel, A Remark on the Existance of finite Graphs, (in Czech) Casopis Pest. Mat. 80 (1955) 477-480.

[7] D. J. Kleitman and D. L. Wang, Algorithms for constructing graphs and digraphs with given valences and factors, Disc. Math. 6 (1973) 79-88.

[8] S. Pirzada, An introduction to Graph Theory, Universities Press, Orient Blackswan (2012) India.

[9] A. R. Rao, An Erdos-Gallai type result on the clique number of a realization of a degree sequence, unpublished.

[10] A. R. Rao, The clique number of a graph with a given degree sequence, Proc. Symposium on

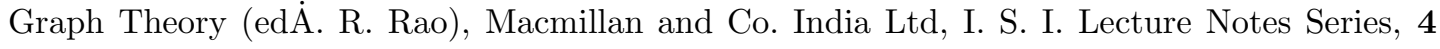
(1979) 251-267.

[11] J. H. Yin and Haikou, A sufficient condition for a sequence to be potentially $S_{r, s}$-graphic, Czech. Math. Journal, 62(137) (2012) 863-867.

\footnotetext{
${ }^{1}$ Department Of Mathematics,

UNIVERSITY OF KASHMIR,

SRINAGAR, KASHMIR, INDIA

E-mail address: pirzadasd@kashmiruniversity.ac.in

E-mail address: sdpirzada@yahoo.co.in

${ }^{2}$ Department of Mathematics,

UNIVERSITY OF KASHMIR,

SRINAGAR, KASHMIR, IndiA

E-mail address: chatbilal@ymail.com
} 